\title{
On the existence of a Feller semigroup with atomic measure in nonlocal boundary condition
}

\author{
Pavel Gurevich
}

\begin{abstract}
The existence of Feller semigroups arising in the theory of multidimensional diffusion processes is studied. An elliptic operator of second order is considered on a plane bounded region $G$. Its domain of definition consists of continuous functions satisfying a nonlocal condition on the boundary of the region. In general, the nonlocal term is an integral of a function over the closure of the region $G$ with respect to a nonnegative Borel measure $\mu(y, d \eta), y \in \partial G$. It is proved that the operator is a generator of a Feller semigroup in the case where the measure is atomic. The smallness of the measure is not assumed.
\end{abstract}

\section{Introduction and Preliminaries}

It was shown in [9, 10] that any one-dimensional diffusion process is related to a strongly continuous contractive nonnegative semigroup (the Feller semigroup) of operators acting on the space of continuous functions. Moreover, a general form of the generator of this semigroup was obtain and all possible boundary conditions defining its domain were described.

In the multidimensional case, a general form of the generator of a Feller semigroup was obtained in [1. It was proved that the generator of a Feller semigroup is an elliptic differential operator of second order (perhaps, degenerated) whose domain of definition consists of continuous functions satisfying a nonlocal boundary condition. The nonlocal term is given by the integral of a function over the closure of a region $G$ with respect to a nonnegative Borel measure $\mu(y, d \eta), y \in \partial G$.

The following problem is unsolved. Given an elliptic differential operator of second order whose domain is defined by a general nonlocal condition (see [1]), whether or not its closure is a generator of a Feller semigroup?

One distinguishes the transversal and nontransversal nonlocal conditions. In the transversal case, the order of nonlocal terms is less than the order of the local terms, whereas these orders coincide in the nontransversal case. The transversal case was considered in [8, 14, 15, 17, 19. A method of the study of the more difficult nontransversal case was developed in papers [2,6, 11, 16]. These works are devoted to obtaining sufficient conditions on the coefficients and the Borel measure (in the nonlocal condition) that ensure the existence of a Feller semigroup.

In [2,11], the authors study the case where the measure $\mu(y, \bar{G})$ (after some normalization) is less than one. In this paper, we investigate nontransversal nonlocal conditions given on the boundary of a plane bounded domain $G$, admitting the "limit case" in which the measure $\mu(y, \bar{G})$ may equal one (it cannot be greater than one [1]). We consider a model case where the measure $\mu(y, d \eta)$ is atomic and vanishes for $y$ from outside of some $\varepsilon$-neighborhood of a set $\mathcal{K} \subset \partial G$ consisting of finitely many points.

By using theorems on the solvability of elliptic equations with nonlocal boundary conditions in the Kondrat'ev weighted spaces [4], asymptotics of solutions near the conjugation points [3] (the 
points of the set $\mathcal{K}$ ), and the maximum principle, we investigate the solvability of nonlocal problems in the spaces of continuous functions (see Secs. 24). Applying these results and the Hille-Iosida theorem, we prove in Sec. 5 that an elliptic operator with the above nonlocal boundary conditions is a generator of a Feller semigroup.

In the conclusion of this section, we remind the notion of a Feller semigroup and its generator and formulate a version of the Hille-Iosida theorem adapted for our purposes.

Let $G \subset \mathbb{R}^{2}$ be a bounded domain with piecewise smooth boundary $\partial G$, and let $X$ be a closed subspace in $C(\bar{G})$ containing at least one nontrivial nonnegative function.

A strongly continuous semigroup of operators $\mathbf{T}_{t}: X \rightarrow X$ is called a Feller semigroup on $X$ if it satisfies the following conditions: 1 . $\left\|\mathbf{T}_{t}\right\| \leq 1, t \geq 0 ; 2$. $\mathbf{T}_{t} u \geq 0$ for all $t \geq 0$ and $u \in X, u \geq 0$.

A linear operator $\mathbf{P}: \mathrm{D}(\mathbf{P}) \subset X \rightarrow X$ is called the (infinitesimal) generator of a strongly continuous semigroup $\left\{\mathbf{T}_{t}\right\}$ if

$$
\mathbf{P} u=\lim _{t \rightarrow+0}(\mathbf{T} u-u) / t, \quad \mathrm{D}(\mathbf{P})=\{u \in X: \text { the limit exists in } X\} .
$$

Theorem 1.1 (the Hille-Iosida theorem, see Theorem 9.3.1 in [17]). 1. Let $\mathbf{P}: \mathrm{D}(\mathbf{P}) \subset X \rightarrow X$ be a generator of a Feller semigroup on $X$. Then the following assertions are true.

(a) The domain $\mathrm{D}(\mathbf{P})$ is dense in $X$.

(b) For each $q>0$ the operator $q \mathbf{I}-\mathbf{P}$ has the bounded inverse $(q \mathbf{I}-\mathbf{P})^{-1}: X \rightarrow X$ and $\left\|(q \mathbf{I}-\mathbf{P})^{-1}\right\| \leq 1 / q$.

(c) The operator $(q \mathbf{I}-\mathbf{P})^{-1}: X \rightarrow X, q>0$, is nonnegative.

2. Conversely, if $\mathbf{P}$ is a linear operator from $X$ to $X$ satisfying condition $(a)$ and there is a constant $q_{1} \geq 0$ such that conditions $(b)$ and $(c)$ hold for $q>q_{1}$, then $\mathbf{P}$ is the generator of a certain Feller semigroup on $X$, which is uniquely determined by $\mathbf{P}$.

\section{Setting of Nonlocal Problems}

Let $G \subset \mathbb{R}^{2}$ be a bounded domain with boundary $\partial G$. Consider a set $\mathcal{K} \subset \partial G$ consisting of finitely

many points. Let $\partial G \backslash \mathcal{K}=\bigcup_{i=1}^{N} \Gamma_{i}$, where $\Gamma_{i}$ are open (in the topology of $\partial G$ ) $C^{\infty}$ curves. Assume that the domain $G$ is a plane angle in some neighborhood of each point $g \in \mathcal{K}$.

For an integer $k \geq 0$, denote by $W_{2}^{k}(G)$ the Sobolev space. By $W_{2, \text { loc }}^{k}(G)$ we denote the set of functions $u$ such that $u \in W_{2}^{k}\left(G^{\prime}\right)$ for any $G^{\prime}, \overline{G^{\prime}} \subset G$.

If $\mathcal{X}$ is a domain in $\mathbb{R}^{2}$, we denote by $C_{0}^{\infty}(\mathcal{X})$ the set of functions infinitely differentiable on $\overline{\mathcal{X}}$ and compactly supported on $\mathcal{X}$. If $\mathcal{M} \subset \mathcal{X}$, we denote by $C_{0}^{\infty}(\overline{\mathcal{X}} \backslash \mathcal{M})$ the set of functions infinitely differentiable on $\overline{\mathcal{X}}$ and compactly supported on $\overline{\mathcal{X}} \backslash \mathcal{M}$.

Along with Sobolev spaces, we will use weighted spaces (the Kondrat'ev spaces). Let $Q=\{y \in$ $\left.\mathbb{R}^{2}: r>0,|\omega|<\omega_{0}\right\}, Q=\left\{y \in \mathbb{R}^{2}: 0<r<d,|\omega|<\omega_{0}\right\}, 0<\omega_{0}<\pi, d>0$, or $Q=G$. We denote by $\mathcal{M}$ the set $\{0\}$ in the first and second cases and the set $\mathcal{K}$ in the third case. Introduce the space $H_{a}^{k}(Q)=H_{a}^{k}(Q, \mathcal{M})$ as the completion of the set $C_{0}^{\infty}(\bar{Q} \backslash \mathcal{M})$ with respect to the norm

$$
\|u\|_{H_{a}^{k}(Q)}=\left(\sum_{|\alpha| \leq k} \int_{Q} \rho^{2(a+|\alpha|-k)}\left|D^{\alpha} u(y)\right|^{2} d y\right)^{1 / 2}
$$


where $a \in \mathbb{R}, k \geq 0$ is an integer, and $\rho=\rho(y)=\operatorname{dist}(y, \mathcal{M})$. For an integer $k \geq 1$, denote by $H_{a}^{k-1 / 2}(\Gamma)=H_{a}^{k-1 / 2}(\Gamma, \mathcal{M})$ the set of traces on a smooth curve $\Gamma \subset \bar{Q}$ (with the infimum-norm).

Let $p_{j k}, p_{j} \in C^{\infty}\left(\mathbb{R}^{2}\right)$ be real-valued functions, and let $p_{j k}=p_{k j}, j, k=1,2$. Consider the differential operator

$$
P u=\sum_{j, k=1}^{2} p_{j k}(y) u_{y_{j} y_{k}}(y)+\sum_{j=1}^{2} p_{j}(y) u_{y_{j}}(y)+p_{0}(y) u(y)
$$

Condition 2.1. 1. There is a constant $c_{0}>0$ such that $\sum_{j, k=1}^{2} p_{j k}(y) \xi_{j} \xi_{k} \geq c_{0}|\xi|^{2}$ for $y \in \bar{G}$ and $\xi=\left(\xi_{1}, \xi_{2}\right) \in \mathbb{R}^{2}$.

2. $p_{0}(y) \leq 0$ for $y \in \bar{G}$.

Introduce the operators corresponding to nonlocal terms supported near the set $\mathcal{K}$. For any set $\mathcal{M}$, we denote its $\varepsilon$-neighborhood by $\mathcal{O}_{\varepsilon}(\mathcal{M})$. Let $\Omega_{i s}\left(i=1, \ldots, N ; s=1, \ldots, S_{i}\right)$ be $C^{\infty}$ diffeomorphisms taking some neighborhood $\mathcal{O}_{i}$ of the curve $\overline{\Gamma_{i} \cap \mathcal{O}_{\varepsilon}(\mathcal{K})}$ to the set $\Omega_{i s}\left(\mathcal{O}_{i}\right)$ in such a way that $\Omega_{i s}\left(\Gamma_{i} \cap \mathcal{O}_{\varepsilon}(\mathcal{K})\right) \subset G$ and $\Omega_{i s}(g) \in \mathcal{K}$ for $g \in \overline{\Gamma_{i}} \cap \mathcal{K}$. Thus, the transformations $\Omega_{i s}$ take the curves $\Gamma_{i} \cap \mathcal{O}_{\varepsilon}(\mathcal{K})$ inside the domain $G$ and the set of their end points $\overline{\Gamma_{i}} \cap \mathcal{K}$ to itself.

Denote by $\Omega_{i s}^{+1}$ the transformation $\Omega_{i s}: \mathcal{O}_{i} \rightarrow \Omega_{i s}\left(\mathcal{O}_{i}\right)$ and by $\Omega_{i s}^{-1}: \Omega_{i s}\left(\mathcal{O}_{i}\right) \rightarrow \mathcal{O}_{i}$ the inverse transformation. The set of points $\Omega_{i_{q} s_{q}}^{ \pm 1}\left(\ldots \Omega_{i_{1} s_{1}}^{ \pm 1}(g)\right) \in \mathcal{K}\left(1 \leq s_{j} \leq S_{i_{j}}, j=1, \ldots, q\right)$ is said to be an orbit of the point $g \in \mathcal{K}$. In other words, the orbit of a point $g \in \mathcal{K}$ is formed by the points (of the set $\mathcal{K}$ ) that can be obtained by consecutively applying the transformations $\Omega_{i_{j} s_{j}}^{ \pm 1}$ to the point $g$.

The set $\mathcal{K}$ consists of finitely many disjoint orbits, which we denote by $\mathcal{K}_{\nu}, \nu=1, \ldots, N_{0}$. Fix an arbitrary orbit $\mathcal{K}_{\nu}$ and assume that it consists of points $1 g_{j}, j=1, \ldots, N_{\nu}$.

Take a sufficiently small number $\varepsilon>0$ such that there exist neighborhoods $\mathcal{O}_{\varepsilon_{1}}\left(g_{j}\right), \mathcal{O}_{\varepsilon_{1}}\left(g_{j}\right) \supset$ $\mathcal{O}_{\varepsilon}\left(g_{j}\right)$, satisfying the following conditions: (1) the domain $G$ is a plane angle in the neighborhood $\mathcal{O}_{\varepsilon_{1}}\left(g_{j}\right) ;(2) \overline{\mathcal{O}_{\varepsilon_{1}}(g)} \cap \overline{\mathcal{O}_{\varepsilon_{1}}(h)}=\varnothing$ for any $g, h \in \mathcal{K}, g \neq h ;(3)$ if $g_{j} \in \overline{\Gamma_{i}}$ and $\Omega_{i s}\left(g_{j}\right)=g_{k}$, then $\mathcal{O}_{\varepsilon}\left(g_{j}\right) \subset \mathcal{O}_{i}$ and $\Omega_{i s}\left(\mathcal{O}_{\varepsilon}\left(g_{j}\right)\right) \subset \mathcal{O}_{\varepsilon_{1}}\left(g_{k}\right)$.

For each point $g_{j} \in \overline{\Gamma_{i}} \cap \mathcal{K}_{\nu}$, we fix a linear transformation $Y_{j}: y \mapsto y^{\prime}\left(g_{j}\right)$ (the composition of the shift by the vector $-\overrightarrow{O g_{j}}$ and rotation) mapping the point $g_{j}$ to the origin in such a way that $Y_{j}\left(\mathcal{O}_{\varepsilon_{1}}\left(g_{j}\right)\right)=\mathcal{O}_{\varepsilon_{1}}(0), Y_{j}\left(G \cap \mathcal{O}_{\varepsilon_{1}}\left(g_{j}\right)\right)=K_{j} \cap \mathcal{O}_{\varepsilon_{1}}(0), Y_{j}\left(\Gamma_{i} \cap \mathcal{O}_{\varepsilon_{1}}\left(g_{j}\right)\right)=\gamma_{j \sigma} \cap \mathcal{O}_{\varepsilon_{1}}(0)(\sigma=1$ or 2$)$, where

$$
K_{j}=\left\{y \in \mathbb{R}^{2}: r>0,|\omega|<\omega_{j}\right\}, \quad \gamma_{j \sigma}=\left\{y \in \mathbb{R}^{2}: r>0, \omega=(-1)^{\sigma} \omega_{j}\right\},
$$

$\omega, r$ are the polar coordinates, $0<\omega_{j}<\pi$. Without loss of generality, we assume that the principal homogeneous part of the operator $P$ at the point $g_{j}$ is the Laplace operator in the new variables $y^{\prime}$.

Condition 2.2. Let $g_{j} \in \overline{\Gamma_{i}} \cap \mathcal{K}_{\nu}$ and $\Omega_{i s}\left(g_{j}\right)=g_{k} \in \mathcal{K}_{\nu}$; then the transformation $Y_{k} \circ \Omega_{i s} \circ Y_{j}^{-1}$ : $\mathcal{O}_{\varepsilon}(0) \rightarrow \mathcal{O}_{\varepsilon_{1}}(0)$ is the composition of rotation and homothety.

Introduce the nonlocal operators $\mathbf{B}_{i} u=\sum_{s=1}^{S_{i}} b_{i s}(y) u\left(\Omega_{i s}(y)\right)$ for $y \in \Gamma_{i} \cap \mathcal{O}_{\varepsilon}(\mathcal{K})$ and $\mathbf{B}_{i} u=0$ for $y \in \Gamma_{i} \backslash \mathcal{O}_{\varepsilon}(\mathcal{K})$, where $b_{i s} \in C^{\infty}\left(\mathbb{R}^{2}\right)$ are real-valued functions, $\operatorname{supp} b_{i s} \subset \mathcal{O}_{\varepsilon}(\mathcal{K})$.

Condition 2.3. The following relations hold:

$$
b_{i s}(y) \geq 0, \quad \sum_{s=1}^{S_{i}} b_{i s}(y) \leq 1, \quad y \in \overline{\Gamma_{i}}
$$

\footnotetext{
${ }^{1}$ The points $g_{j}$ and other objects (see below) related to the orbit $\mathcal{K}_{\nu}$ depend on $\nu$. To avoid clumsy notation, we do not explicitly indicate this dependence.
} 


$$
\sum_{s=1}^{S_{i}} b_{i s}(g)+\sum_{s=1}^{S_{j}} b_{j s}(g)<2, \quad g \in \overline{\Gamma_{i}} \cap \overline{\Gamma_{j}} \subset \mathcal{K}, \quad \text { if } i \neq j \text { and } \overline{\Gamma_{i}} \cap \overline{\Gamma_{j}} \neq \varnothing .
$$

We will study the nonlocal elliptic problem

$$
P u-q u=f(y), y \in G ;\left.\quad u\right|_{\Gamma_{i}}-\mathbf{B}_{i} u=0, y \in \Gamma_{i}, i=1, \ldots, N,
$$

where $q \geq 0$, and the same problem with nonhomogeneous nonlocal conditions. To consider problem (2.5) in spaces of continuous functions, we preliminarily study it in the weighted spaces.

In the sequel, we need norms in weighted spaces depending on the parameter $q>0$. Set

$$
\begin{gathered}
\|u\|_{H_{a}^{k}(G)}=\left(\|u\|_{H_{a}^{k}(G)}^{2}+q^{k}\|u\|_{H_{a}^{0}(G)}^{2}\right)^{1 / 2}, \quad k \geq 0, \\
\|v\|_{H_{a}^{k-1 / 2}\left(\Gamma_{i}\right)}=\left(\|v\|_{H_{a}^{k-1 / 2}\left(\Gamma_{i}\right)}^{2}+q^{k-1 / 2}\|v\|_{H_{a}^{0}\left(\Gamma_{i}\right)}^{2}\right)^{1 / 2}, \quad k \geq 1,
\end{gathered}
$$

where $\|v\|_{H_{a}^{0}\left(\Gamma_{i}\right)}=\left(\int_{\Gamma_{i}} \rho^{2 a}|v(y)|^{2} d \Gamma\right)^{2}$. We also consider the following spaces:

- $\mathcal{H}_{a}^{k+3 / 2}(\partial G)=\prod_{i=1}^{N} H_{a}^{k+3 / 2}\left(\Gamma_{i}\right),\|\psi\|_{\mathcal{H}_{a}^{k+3 / 2}(\partial G)}=\left(\sum_{i=1}^{N}\left\|\psi_{i}\right\|_{H_{a}^{k+3 / 2}\left(\Gamma_{i}\right)}^{2}\right)^{1 / 2}, \psi=\left\{\psi_{i}\right\}$,

- $\mathcal{H}_{a}^{k}(G, \partial G)=H_{a}^{k}(G) \times \mathcal{H}_{a}^{k+3 / 2}(\partial G),\|(f, \psi)\|_{\mathcal{H}_{a}^{k}(G, \partial G)}=\left(\|f\|_{H_{a}^{k}(G)}^{2}+\|\psi\|_{\mathcal{H}_{a}^{k+3 / 2}(\partial G)}^{2}\right)^{1 / 2}$.

Consider the bounded operator

$$
\begin{gathered}
\mathbf{L}(q): H_{k+1-\delta}^{k+2}(G) \rightarrow \mathcal{H}_{k+1-\delta}^{k}(G, \partial G), \\
\mathbf{L}(q) u=\left\{P u-q u,\left.u\right|_{\Gamma_{i}}-\mathbf{B}_{i} u\right\}, \quad q \geq 0 .
\end{gathered}
$$

We prove the following theorem in Sec. [3.

Theorem 2.1. Let Conditions 2.12 .3 hold, and let $k \geq 0$ be fixed. Then there exists a number $\delta_{1}>0$ possessing the following property: for any $\delta \in\left[0, \delta_{1}\right]$, there is a number $q_{1}=q_{1}(\delta)>0$ such that the operator $\mathbf{L}(q)$ has a bounded inverse for $q \geq q_{1}$ and

$$
c\|\mathbf{L}(q) u\|_{\mathcal{H}_{k+1-\delta}^{k}(G, \partial G)} \leq\|u\|_{H_{k+1-\delta}^{k+2}(G)} \leq C\|\mathbf{L}(q) u\|_{\mathcal{H}_{k+1-\delta}^{k}(G, \partial G)}, \quad q \geq q_{1},
$$

where $c, C>0$ do not depend on $u$ and $q$.

\section{Nonlocal Problems in Weighted Spaces}

We fix an arbitrary orbit $\mathcal{K}_{\nu}$ and assume that it consists of points $g_{j}, j=1, \ldots, N_{\nu}$. Denote by $u_{j}(y)$ the function $u(y)$ for $y \in \mathcal{O}_{\varepsilon_{1}}\left(g_{j}\right)$. If $g_{j} \in \overline{\Gamma_{i}}, y \in \mathcal{O}_{\varepsilon}\left(g_{j}\right)$, and $\Omega_{i s}(y) \in \mathcal{O}_{\varepsilon_{1}}\left(g_{k}\right)$, then denote by $u_{k}\left(\Omega_{i s}(y)\right)$ the function $u\left(\Omega_{i s}(y)\right)$. In this case, nonlocal problem (2.5) takes the following form in the $\varepsilon$-neighborhood of the orbit $\mathcal{K}_{\nu}$ :

$$
\begin{gathered}
P u_{j}-q u_{j}=f(y), \quad y \in \mathcal{O}_{\varepsilon}\left(g_{j}\right) \cap G, \\
u_{j}(y)-\sum_{s=1}^{S_{i}} b_{i s}(y) u_{k}\left(\Omega_{i s}(y)\right)=0, \quad y \in \mathcal{O}_{\varepsilon}\left(g_{j}\right) \cap \Gamma_{i}, i \in\left\{1 \leq i \leq N: g_{j} \in \overline{\Gamma_{i}}\right\}, j=1, \ldots, N_{\nu} .
\end{gathered}
$$


Let $y \mapsto y^{\prime}\left(g_{j}\right)$ be the change of variables described in Sec. 2, and let $K_{j}$ and $\gamma_{j \sigma}$ be the sets defined in (2.2). Set $K_{j}^{\varepsilon}=K_{j} \cap \mathcal{O}_{\varepsilon}(0), \gamma_{j \sigma}^{\varepsilon}=\gamma_{j \sigma} \cap \mathcal{O}_{\varepsilon}(0)$. Introduce the functions $U_{j}\left(y^{\prime}\right)=u\left(y\left(y^{\prime}\right)\right)$ and $F_{j}\left(y^{\prime}\right)=f\left(y\left(y^{\prime}\right)\right)$ for $y^{\prime} \in K_{j}^{\varepsilon}$, where $\sigma=1(\sigma=2)$ if the transformation $y \mapsto y^{\prime}\left(g_{j}\right)$ takes $\Gamma_{i}$ to the side $\gamma_{j 1}\left(\gamma_{j 2}\right)$ of the angle $K_{j}$. Denote $y^{\prime}$ by $y$ again. Then, by virtue of Condition 2.2 , problem (2.5) takes the form

$$
P_{j}\left(y, D_{y}\right) U_{j}-q U_{j}=F_{j}(y), y \in K_{j}^{\varepsilon} ; \quad U_{j}(y)-\sum_{k=1}^{N_{\nu}} \sum_{s=1}^{S_{j \sigma k}} B_{j \sigma k s}(y) U_{k}\left(\mathcal{G}_{j \sigma k s} y\right)=0, y \in \gamma_{j \sigma}^{\varepsilon} .
$$

Here $P_{j}\left(y, D_{y}\right)$ is a second-order elliptic differential operator with real-valued $C^{\infty}$ coefficients such that the principal homogeneous part of $P_{j}\left(0, D_{y}\right)$ is the Laplace operator $\Delta ; B_{j \sigma k s}(y)$ are smooth functions; $\mathcal{G}_{j \sigma k s}$ is an operator of rotation by an angle $\omega_{j \sigma k s}$ and homothety with a coefficient $\chi_{j \sigma k s}>0$ such that $\left|(-1)^{\sigma} \omega_{j}+\omega_{j \sigma k s}\right|<\omega_{k}$.

Following [4], we freeze the coefficients of problem (3.1) at the point $y=0$, replace the operators $P_{j}\left(0, D_{y}\right)$ by their principal homogeneous parts, and set $q=1$. Thus, we consider the following problem:

$$
\Delta U_{j}-U_{j}=F_{j}(y), y \in K_{j} ; \quad \mathcal{B}_{j \sigma} U \equiv U_{j}(y)-\sum_{k=1}^{N_{\nu}} \sum_{s=1}^{S_{j \sigma k}} b_{j \sigma k s} U_{k}\left(\mathcal{G}_{j \sigma k s} y\right)=0, y \in \gamma_{j \sigma}
$$

where $U=\left(U_{1}, \ldots, U_{N_{\nu}}\right)$ and $b_{j \sigma k s}=B_{j \sigma k s}(0)$. It follows from Condition 2.3 that

$$
b_{j \sigma k s} \geq 0, \quad \sum_{k=1}^{N_{\nu}} \sum_{s=1}^{S_{j \sigma k}} b_{j \sigma k s} \leq 1, \quad \sum_{k=1}^{N_{\nu}}\left(\sum_{s=1}^{S_{j 1 k}} b_{j 1 k s}+\sum_{s=1}^{S_{j 2 k}} b_{j 2 k s}\right)<2 .
$$

Problem (3.2) should be studied in weighted spaces with nonhomogeneous weight (cf. [4]). Denote by $E_{a}^{k}\left(K_{j}\right)$ the completion of the set $C_{0}^{\infty}\left(\overline{K_{j}} \backslash\{0\}\right)$ with respect to the norm

$$
\|v\|_{E_{a}^{k}\left(K_{j}\right)}=\left(\sum_{|\alpha| \leq k} \int_{K_{j}}|y|^{2 a}\left(|y|^{2(|\alpha|-k)}+1\right)\left|D^{\alpha} v(y)\right|^{2} d y\right)^{1 / 2}
$$

where $k \geq 0$ is an integer and $a \in \mathbb{R}$. Denote by $E_{a}^{k-1 / 2}\left(\gamma_{j \sigma}\right)(k \geq 1$ is an integer $)$ the space of traces on $\gamma_{j \sigma}$ (with the infimum-norm). Introduce the spaces of vector-valued functions

$$
\mathcal{E}_{a}^{k+2}(K)=\prod_{j=1}^{N_{\nu}} E_{a}^{k+2}\left(K_{j}\right), \quad \mathcal{E}_{a}^{k}(K, \gamma)=\prod_{j=1}^{N_{\nu}}\left(E_{a}^{k}\left(K_{j}\right) \times \prod_{\sigma=1,2} E_{a}^{k+3 / 2}\left(\gamma_{j \sigma}\right)\right)
$$

Consider the operator $\mathcal{L}: \mathcal{E}_{1-\delta}^{2}(K) \rightarrow \mathcal{E}_{1-\delta}^{0}(K, \gamma)$ given by $\mathcal{L} U=\left\{\Delta U_{j}-U_{j}, \mathcal{B}_{j \sigma} U\right\}$.

Our aim is to prove that the operator $\mathcal{L}$ is an isomorphism for all sufficiently small $\delta \geq 0$. To this end, we consider the analytic operator-valued function

$$
\begin{gathered}
\tilde{\mathcal{L}}(\lambda): \prod_{j=1}^{N_{\nu}} W_{2}^{2}\left(-\omega_{j}, \omega_{j}\right) \rightarrow \prod_{j=1}^{N_{\nu}}\left(L_{2}\left(-\omega_{j}, \omega_{j}\right) \times \mathbb{C}^{2}\right) \\
\tilde{\mathcal{L}}(\lambda) \varphi=\left\{\varphi_{j}^{\prime \prime}-\lambda^{2} \varphi_{j}, \varphi_{j}\left((-1)^{\sigma} \omega_{j}\right)-\sum_{k, s}\left(\chi_{j \sigma k s}\right)^{i \lambda} b_{j \sigma k s} \varphi_{k}\left((-1)^{\sigma} \omega_{j}+\omega_{j \sigma k s}\right)\right\} .
\end{gathered}
$$


Lemma 3.1. Let Conditions 2.12 .3 hold. Then the line $\operatorname{Im} \lambda=0$ contains no eigenvalues of $\tilde{\mathcal{L}}(\lambda)$.

Proof. 1. We assume that $\lambda_{0} \neq 0$ (the case $\lambda_{0}=0$ is analogous but simpler) is an eigenvalue of $\tilde{\mathcal{L}}(\lambda)$ and $\lambda_{0}$ is a real number. Let $\varphi(\omega)$ be the corresponding eigenvector. We represent it in the form $\varphi(\omega)=\varphi^{1}(\omega)+i \varphi^{2}(\omega)$, where $\varphi^{1}(\omega)$ and $\varphi^{2}(\omega)$ are real-valued $C^{\infty}$ functions. It is easy to see that the function $U=r^{i \lambda_{0}} \varphi(\omega)=e^{i \lambda_{0} \ln r} \varphi(\omega)$ is a solution of the following problem:

$$
\Delta U_{j}=0, y \in K_{j} ; \quad \mathcal{B}_{j \sigma} U=0, y \in \gamma_{j \sigma} .
$$

We represent the function $U$ in the form $U=V+i W$, where $V=\cos \left(\lambda_{0} \ln r\right) \varphi^{1}(\omega)-\sin \left(\lambda_{0} \ln r\right) \varphi^{2}(\omega)$, $W=\cos \left(\lambda_{0} \ln r\right) \varphi^{2}(\omega)+\sin \left(\lambda_{0} \ln r\right) \varphi^{1}(\omega)$. Since the coefficients in (3.4) are real, it follows that $V$ (as well as $W$ ) is a solution of the problem

$$
\Delta V_{j}=0, y \in K_{j} ; \quad \mathcal{B}_{j \sigma} V=0, y \in \gamma_{j \sigma}
$$

Denote $M=\max _{j=1, \ldots, N_{\nu}} \sup _{y \in K_{j}}\left|V_{j}(y)\right|$. We claim that $M=0$. Assume the contrary: $M>0$.

2. If $\left|V_{j}\left(y^{0}\right)\right|=M$ for some $j$ and $y^{0} \in K_{j}$, then $V_{j}(y) \equiv M$ by the maximum principle, and the nonlocal conditions in (3.5) imply

$$
M=\left|V_{j}\left(y^{0}\right)\right|=\left|V_{j}\right|_{\gamma_{j \sigma}} \mid \leq M \sum_{k, s} b_{j \sigma k s}, \quad \sigma=1,2 .
$$

However, $0 \leq \sum_{k, s} b_{j \sigma k s}<1$ for $\sigma=1$ or 2 due to conditions (3.3), which contradicts (3.6).

3. Let $\left|V_{j}\left(y_{0}\right)\right|=M$ for some $j, \sigma=1$ or 2 , and $y_{0} \in \gamma_{j \sigma}$. In this case, taking into account (3.3), we again deduce from the nonlocal conditions in (3.5) that

$$
M=\left|V_{j}\left(y^{0}\right)\right| \leq \sum_{k, s} b_{j \sigma k s}\left|V_{k}\left(\mathcal{G}_{j \sigma k s} y^{0}\right)\right| \leq M
$$

for $\sigma=1$ or 2. Therefore, the inequalities in (3.7) reduce to equalities, and we see that $\sum_{k, s} b_{j \sigma k s}=1$ and $\left|V_{k}\left(\mathcal{G}_{j \sigma k s} y^{0}\right)\right|=M$ for at least one pair $(k, s)$. However, this contradicts what has been already proved, since $\mathcal{G}_{j \sigma k s} y^{0} \in K_{k}$.

4. Finally, we assume that there is a sequence $\left\{y^{s}\right\}_{s=1}^{\infty} \subset K_{j}$ such that $\left|V_{j}\left(y^{s}\right)\right| \rightarrow M$ for some $j$ as $\left|y^{s}\right| \rightarrow 0$ or $\left|y^{s}\right| \rightarrow \infty$.

We note that the function $V_{j}$ is periodic with respect to $\ln r$, i.e., the function $V_{j}$ is completely defined by its values on the set $\hat{K}_{j}=\overline{K_{j}} \cap\left\{1 \leq r \leq e^{2 \pi /\left|\lambda_{0}\right|}\right\}$.

Since the set $\hat{K}_{j}$ is a compact, there is a sequence $\left\{\hat{y}^{s}\right\}_{s=1}^{\infty} \subset \hat{K}_{j}$ such that $\left|V_{j}\left(\hat{y}^{s}\right)\right| \rightarrow M$ as $\hat{y}^{s} \rightarrow \hat{y}$, where $\hat{y} \in \hat{K}_{j}$. It follows from the continuity of the function $V_{j}(y)$ on the compact $\hat{K}_{j}$ that $\left|V_{j}(\hat{y})\right|=M$. However, this is impossible due to what has been proved above.

5. It follows from items $1-4$ that $M=0$, hence $V=0$, i.e., $\varphi^{1}(\omega)=\varphi^{2}(\omega)=0$.

Lemma 3.2. Let Conditions 2.1$] 2.3$ hold. Then the operator $\mathcal{L}: \mathcal{E}_{1}^{2}(K) \rightarrow \mathcal{E}_{1}^{0}(K, \gamma)$ is an isomorphism.

Proof. 1. First, we show that the operator $\mathcal{L}: \mathcal{E}_{1}^{2}(K) \rightarrow \mathcal{E}_{1}^{0}(K, \gamma)$ has the Fredholm property and ind $\mathcal{L}=0$. Consider the family of operators $\mathcal{L}_{t}: \mathcal{E}_{1}^{2}(K) \rightarrow \mathcal{E}_{1}^{0}(K, \gamma)$ given by $\mathcal{L}_{t} U=\left\{\Delta U_{j}-\right.$ $\left.U_{j},\left.U_{j}\right|_{\gamma_{j \sigma}}-\left.t \sum_{k, s} b_{j \sigma k s} U_{k}\left(\mathcal{G}_{j \sigma k s} y\right)\right|_{\gamma_{j \sigma}}\right\}, 0 \leq t \leq 1$. Similarly to the operator $\tilde{\mathcal{L}}(\lambda)$, we introduce the operators $\tilde{\mathcal{L}}_{t}(\lambda)$. By Lemma 3.1 , the operators $\tilde{\mathcal{L}}_{t}(\lambda)$ have no eigenvalues on the $\operatorname{line} \operatorname{Im} \lambda=0$. 
Therefore, the operators $\mathcal{L}_{t}$ have the Fredholm property due to Theorem 9.1 in [12]. By using the homotopy stability of the index of Fredholm operators, we obtain ind $\mathcal{L}_{t}=$ const for $t \in[0,1]$. Since the local operator $\mathcal{L}_{0}$ is an isomorphism (see, e.g., Sec. 10.3 in [12]), it follows that ind $\mathcal{L}=$ ind $\mathcal{L}_{0}=0$.

2. It remains to prove that $\operatorname{dim} \operatorname{ker} \mathcal{L}=0$. Let $U \in \mathcal{E}_{1}^{2}(K)$ be a real-valued solution of the problem

$$
\Delta U_{j}=U_{j}, y \in K_{j} ; \quad \mathcal{B}_{j \sigma} U=0, y \in \gamma_{j \sigma} .
$$

Due to the interior regularity theorem, the functions $U_{j}$ are infinitely differentiable in $K_{j}$. Let us prove that $U_{j}$ are continuous on $\overline{K_{j}}$.

Since the line $\operatorname{Im} \lambda=0$ contains no eigenvalues of $\tilde{\mathcal{L}}(\lambda)$, it follows from [7] that there is a number $\delta \in[0,1]$ such that the strip $-1-\delta \leq \operatorname{Im} \lambda \leq 0$ contains finitely many eigenvalues $\left\{\lambda_{k}\right\}$ of $\tilde{\mathcal{L}}(\lambda)$ and $-1-\delta<\operatorname{Im} \lambda_{k}<0$. Taking into account that $U_{j} \in E_{1}^{2}\left(K_{j}\right) \subset H_{1}^{2}\left(K_{j}\right)$ is a solution of problem (3.8) with the right-hand sides $U_{j} \in E_{1}^{2}\left(K_{j}\right) \subset H_{-\delta}^{0}\left(K_{j}\right)$ and applying Theorem 2.2 in [3] (about the asymptotics of solutions for nonlocal problems), we obtain

$$
U=\sum_{k} \sum_{q=1}^{J_{k}} \sum_{m=0}^{\varkappa_{q k}-1} c_{k}^{(m, q)} W_{k}^{(m, q)}+U^{\prime}, \quad W_{k}^{(m, q)}(\omega, r)=r^{i \lambda_{k}} \sum_{l=0}^{m} \frac{1}{l !}(i \ln r)^{l} \varphi_{k}^{(m-l, q)}(\omega),
$$

where $\varphi_{k}^{(0, q)}, \ldots, \varphi_{k}^{\left(\varkappa_{q k}-1, q\right)} \in \prod_{j} C^{\infty}\left(\left[-\omega_{j}, \omega_{j}\right]\right)$ is the Jordan chain corresponding to the eigenvalue $\lambda_{k}$, $c_{k}^{(m, q)}$ are constants, and $U_{j}^{\prime} \in H_{-\delta}^{2}\left(K_{j}\right)$. Thus, using the Sobolev embedding theorem, we see that the functions $U_{j}$ are continuous on $\overline{K_{j}}$ and $U_{j}(0)=0$.

Furthermore, we claim that

$$
\left|U_{j}(y)\right| \rightarrow 0 \quad \text { as } \quad|y| \rightarrow \infty
$$

Indeed, since $U \in \mathcal{E}_{1}^{2}(K)$, it follows that $U \in \mathcal{E}_{1}^{0}(K)$. Combining this with the fact that $U$ is a solution of homogeneous problem (3.8) and applying Theorem 3.2 in [12], we obtain $U \in \mathcal{E}_{3}^{2}(K)$. Fixing an arbitrary $a \geq 1$ and repeating these arguments finitely many times, we have $U \in \mathcal{E}_{a}^{2}(K)$. Setting $V(\omega, r)=U\left(\omega, r^{-1}\right)$ and using the Sobolev embedding theorem and the fact that $a$ can be arbitrarily large, we see that the functions $V_{j}(y)$ are continuous at the origin and $\left|V_{j}(y)\right| \rightarrow 0$ as $|y| \rightarrow 0$. This implies (3.10)

3. Set $M=\max _{j=1, \ldots, N_{\nu}} \sup _{y \in \overline{K_{j}}}\left|U_{j}(y)\right|$. We claim that $M=0$. Assume the contrary; let $M>0$. Due to the above properties of $U_{j}$, each of the functions $\left|U_{j}(y)\right|$ achieves its maximum at some point $y_{0} \in \overline{K_{j}} \backslash\{0\}$. If $\left|U_{j}\left(y_{0}\right)\right|=M$ for some $j$ and $y_{0} \in K_{j}$, then $U_{j}(y) \equiv$ const by the maximum principle. In this case, using the equation in (3.8), we obtain $M \equiv\left|U_{j}\right|=\left|\Delta U_{j}\right|=0$.

If $\left|U_{j}\left(y_{0}\right)\right|=M$ for $y_{0} \in \gamma_{j \sigma}$, where $\sigma=1$ or 2 , then, using the nonlocal conditions in (3.8) and inequalities (3.3), we obtain

$$
M=\left|U_{j}\left(y_{0}\right)\right| \leq \sum_{k, s} b_{j \sigma k s}\left|U_{k}\left(\mathcal{G}_{j \sigma k s} y_{0}\right)\right| \leq M
$$

Thus, the inequalities in (3.11) becomes the equalities, which implies $\sum_{k, s} b_{j \sigma k s}=1$ and $\left|U_{k}\left(\mathcal{G}_{j \sigma k s} y_{0}\right)\right|=$ $M$ for at least one pair $(k, s)$. However, $\mathcal{G}_{j \sigma k s} y_{0} \in K_{k}$, which is impossible by what has been proved above.

Corollary 3.1. Let Conditions 2.122 .3 hold. Then there exists a number $\delta_{1}>0$ such that the operator $\mathcal{L}: \mathcal{E}_{k+1-\delta}^{k+2}(K) \rightarrow \mathcal{E}_{k+1-\delta}^{k}(K, \gamma), k=0,1,2, \ldots$, is an isomorphism for $\delta \in\left[0, \delta_{1}\right]$. 
Proof. By Lemma 3.2, the operator $\mathcal{L}: \mathcal{E}_{1}^{2}(K) \rightarrow \mathcal{E}_{1}^{0}(K, \gamma)$ is an isomorphism. On the other hand, it follow from Lemma 3.1 and from the discreteness of the spectrum of $\tilde{L}(\lambda)$ (see [7]), there exists a number $\delta_{1}>0$ such that the strip $-\delta_{1} \leq \operatorname{Im} \lambda \leq 0$ contains no eigenvalues of $\tilde{\mathcal{L}}(\lambda)$. Similarly to Proposition 2.8 in [5, Chap. 8], one can show that the operator $\mathcal{L}: \mathcal{E}_{1-\delta}^{2}(K) \rightarrow \mathcal{E}_{1-\delta}^{0}(K, \gamma)$ is an isomorphism for $\delta \in\left[0, \delta_{1}\right]$. Due to Theorems 9.2 and 9.3 in [12], the operator $\mathcal{L}: \mathcal{E}_{k+1-\delta}^{k+2}(K) \rightarrow$ $\mathcal{E}_{k+1-\delta}^{k}(K, \gamma)$ is also an isomorphism.

Proof of Theorem 2.1. The required assertion follows from Theorem 8.1 in [4] and Corollary 3.1.

\section{Nonlocal Problems in Spaces of Continuous Functions}

In what follows, we assume that a number $\delta \in[0,1]$ is fixed in such a way that neither the strip $-\delta \leq \operatorname{Im} \lambda \leq 0$ nor the line $\operatorname{Im} \lambda=-1-\delta$ contain an eigenvalue of $\tilde{\mathcal{L}}(\lambda)$. The existence of such a number follows from Lemma 3.1 and from the discreteness of the spectrum of $\tilde{L}(\lambda)$ (see [7]).

Let $q_{1}$ be the number occurring in Theorem 2.1. First, we construct an analog of the barrier function for nonlocal problems. Consider the following auxiliary problem:

$$
P v-q_{1} v=0, y \in G ;\left.\quad v\right|_{\Gamma_{i}}-\mathbf{B}_{i} v=1, y \in \Gamma_{i}, i=1, \ldots, N .
$$

Lemma 4.1. Let Conditions 2.12 .3 hold. Then problem (4.1) admits a bounded solution $v \in$ $C^{\infty}(\bar{G} \backslash \mathcal{K})$ such that $\inf _{y \in \bar{G} \backslash \mathcal{K}} v(y)>0$.

Proof. 1. We fix an arbitrary orbit $\mathcal{K}_{\nu}$ and consider the model problem

$$
\Delta W_{j}^{1}=0, y \in K_{j}^{\varepsilon} ; \quad W_{j}^{1}(y)-\sum_{k, s} b_{j \sigma k s} W_{k}^{1}\left(\mathcal{G}_{j \sigma k s} y\right)=1, y \in \gamma_{j \sigma}^{\varepsilon} .
$$

Let us search a solution of problem (4.2) in the form

$$
W_{j}^{1}=\varphi_{j}(\omega), \quad|\omega|<\omega_{j}, j=1, \ldots, N_{\nu}
$$

Clearly, the functions $\varphi_{1}(\omega), \ldots, \varphi_{N_{\nu}}(\omega)$ must satisfy the relations

$$
\varphi_{j}^{\prime \prime}(\omega)=0,|\omega|<\omega_{j} ; \quad \varphi_{j}\left((-1)^{\sigma} \omega_{j}\right)-\sum_{k, s} b_{j \sigma k s} \varphi_{k}\left((-1)^{\sigma} \omega_{j}+\omega_{j \sigma k s}\right)=1
$$

or, equivalently, $\tilde{\mathcal{L}}(0) \varphi=\left\{\tilde{F}_{j}, \tilde{F}_{j \sigma}\right\}, \tilde{F}_{j}=0, \tilde{F}_{j \sigma}=1$. Due to Lemma 3.1, the number $\lambda=0$ is not an eigenvalue of $\tilde{\mathcal{L}}(\lambda)$. Since the operator $\tilde{\mathcal{L}}(\lambda)$ has the Fredhom property and its index equals zero [7], there exists a unique (real-valued) solution $\varphi \in \prod_{j} C^{\infty}\left(\left[-\omega_{j}, \omega_{j}\right]\right)$ of problem (4.4). Clearly, $\varphi_{j}(\omega)$ are linear functions. Using the nonlocal conditions in (4.4) and relations (3.3), one can check that $\varphi_{j}(\omega)>0$ for $\omega \in\left[-\omega_{j}, \omega_{j}\right]$.

2. Consider a function $\xi \in C^{\infty}\left(\mathbb{R}^{2}\right)$ such that $\xi(y)=1$ for $y \in \mathcal{O}_{\varepsilon / 2}(\mathcal{K})$ and $\operatorname{supp} \xi \subset \mathcal{O}_{\varepsilon}(\mathcal{K})$.

Let us search the solution $v$ of the original problem (4.1) in the form

$$
v(y)=w^{1}(y)+v^{1}(y), \quad y \in G,
$$

where $w^{1}(y)=\xi(y) W_{j}^{1}\left(y^{\prime}(y)\right), y \in \mathcal{O}_{\varepsilon}\left(g_{j}\right), g_{j} \in \mathcal{K}_{\nu}, y^{\prime} \mapsto y\left(g_{j}\right)$ is the transformation inverse to the transformation $y \mapsto y^{\prime}\left(g_{j}\right)$ from Sec. 2, and the function $w^{1}$ is extended by zero to $G \backslash \mathcal{O}_{\varepsilon}(\mathcal{K})$; the function $v^{1}$ is unknown. 
It follows from relations (4.1) and (4.5) that the function $v^{1}$ satisfies the relations

$$
P v^{1}-q_{1} v^{1}=f^{1}(y), y \in G ;\left.\quad v^{1}\right|_{\Gamma_{i}}-\mathbf{B}_{i} v^{1}=f_{i}^{1}(y), y \in \Gamma_{i},
$$

where

$$
f^{1}=-P w^{1}+q_{1} w^{1}, \quad f_{i}^{1}=1-\left.w^{1}\right|_{\Gamma_{i}}+\left.\mathbf{B}_{i} w^{1}\right|_{\Gamma_{i}} .
$$

Set $V_{j}^{1}\left(y^{\prime}\right)=v^{1}\left(y\left(y^{\prime}\right)\right), F_{j}\left(y^{\prime}\right)=f^{1}\left(y\left(y^{\prime}\right)\right)$, and $F_{j \sigma}\left(y^{\prime}\right)=f_{i}^{1}\left(y\left(y^{\prime}\right)\right), y^{\prime} \in K_{j}^{\varepsilon}$, where $y \mapsto y^{\prime}\left(g_{j}\right)$ is the transformation from Sec. 2, $g_{j} \in \mathcal{K}_{\nu} \cap \overline{\Gamma_{i}}$. Denote $y^{\prime}$ by $y$ again. Then, due to (4.2) and (4.7), we have

$$
F_{j}(y)=\left(\Delta-P_{j}\left(y, D_{y}\right)\right) W_{j}^{1}+q_{1} W_{j}^{1}, \quad F_{j \sigma}(y)=\sum_{k, s}\left(B_{j \sigma k s}(y)-b_{j \sigma k s}\right) W_{k}^{1}\left(\mathcal{G}_{j \sigma k s} y\right), \quad y \in K_{j}^{\varepsilon / 2},
$$

where $P_{j}\left(y, D_{y}\right)$ and $B_{j \sigma k s}(y)$ are the same as in (3.1).

Using the facts the the principal homogeneous part of the operator $P_{j}\left(0, D_{y}\right)$ is the Laplace operator and $B_{j \sigma k s}(0)=b_{j \sigma k s}$ and applying the Taylor formula, we deduce from representation (4.3) and relations (4.8) that $F_{j} \in H_{k+1-\delta}^{k}\left(K_{j}^{\varepsilon / 2}\right)$ and $F_{j \sigma} \in H_{k+1-\delta}^{k+3 / 2}\left(\gamma_{j \sigma}^{\varepsilon / 2}\right)$, i.e., $\left\{f^{1}, f_{i}^{1}\right\} \in \mathcal{H}_{k+1-\delta}^{k}(G, \partial G)$. Therefore, by Theorem 2.1, there is a unique solution $v^{1} \in H_{k+1-\delta}^{k+2}(G)$ of problem (4.6). Since $k \geq 0$ is arbitrary, it follows from the Sobolev embedding theorem that the function $v$ given by (4.5) belongs to $C^{\infty}(\bar{G} \backslash \mathcal{K})$. Clearly, it is a solution of the original problem (4.1).

3. Let us prove that $v^{1} \in C(\bar{G})$ and $v^{1}(y)=0$ for $y \in \mathcal{K}$. Due to (4.6), the functions $V_{j}^{1}(y)$ satisfy the following relations:

$$
\Delta V_{j}^{1}=F_{j}^{1}(y)+F_{j}(y), y \in K_{j}^{\varepsilon / 2} ; \quad V_{j}^{1}(y)-\sum_{k, s} b_{j \sigma k s} V_{k}^{1}\left(\mathcal{G}_{j \sigma k s} y\right)=F_{j \sigma}^{1}(y)+F_{j \sigma}(y), y \in \gamma_{j \sigma}^{\varepsilon / 2},
$$

where $F_{j}^{1}=\left(\Delta-P_{j}\left(y, D_{y}\right)\right) V_{j}^{1}+q_{1} V_{j}^{1}$ and $F_{j \sigma}^{1}=\sum_{k, s}\left(B_{j \sigma k s}(y)-b_{j \sigma k s}\right) V_{k}^{1}\left(\mathcal{G}_{j \sigma k s} y\right)$.

Using the facts that the principal homogeneous part of the operator $P_{j}\left(0, D_{y}\right)$ is the Laplace operator and $B_{j \sigma k s}(0)=b_{j \sigma k s}$ and applying the Taylor formula once more, we represent the righthand sides of problem (4.9) as follows:

$$
F_{j}^{1}+F_{j}=F_{j}^{1}+F_{j}^{2}+r^{-1} \psi_{j}(\omega), \quad F_{j \sigma}^{1}+F_{j \sigma}=F_{j \sigma}^{1}+F_{j \sigma}^{2}+\psi_{j \sigma} r,
$$

where $\psi_{j} \in C^{\infty}\left(\left[-\omega_{j}, \omega_{j}\right]\right), F_{j}^{1}+F_{j}^{2} \in H_{-\delta}^{0}\left(K_{j}^{\varepsilon / 2}\right)$ and $\psi_{j \sigma} \in \mathbb{R}, F_{j \sigma}^{1}+F_{j \sigma}^{2} \in H_{-\delta}^{3 / 2}\left(\gamma_{j \sigma}^{\varepsilon / 2}\right)$.

To obtain the asymptotics of the functions $V_{j}^{1}$, we denote by $\left\{\lambda_{k}\right\}$ a (finite) set of eigenvalues of $\tilde{\mathcal{L}}(\lambda)$ lying in the strip $-1-\delta<\operatorname{Im} \lambda<-\delta$. Then Theorem 2.2 in [3] and Lemma 4.3 in [3] applied to problem (4.9) with right-hand side (4.10) imply that

$$
V^{1}=r \sum_{l=0}^{\varkappa} \frac{1}{l !}(i \ln r)^{l} u^{(l)}(\omega)+\sum_{k} \sum_{q=1}^{J_{k}} \sum_{m=0}^{\varkappa_{q k}-1} c_{k}^{(m, q)} W_{k}^{(m, q)}+V^{2}, \quad y \in K_{j}^{\varepsilon / 2},
$$

where $u^{(l)} \in \prod_{j} C^{\infty}\left(\left[-\omega_{j}, \omega_{j}\right]\right)$, the functions $W_{k}^{(m, q)}$ are of the same form as in (3.9), $c_{k}^{(m, q)}$ are some constants, and $V_{j}^{2} \in H_{-\delta}^{2}\left(K_{j}^{\varepsilon / 2}\right)$. The asymptotic formula (4.11) and the Sobolev embedding theorem imply that $V_{j}^{1} \in C\left(\overline{K_{j}^{\varepsilon / 2}}\right)$ and $V_{j}^{1}(0)=0$. Therefore, $v^{1} \in C(\bar{G})$ and $v^{1}(0)=0$ for $y \in \mathcal{K}$. In particular, this means that the function $v=v^{1}+w^{1}$ is bounded.

4. It remains to show that $m>0$, where $m=\inf _{y \in \bar{G} \backslash \mathcal{K}} v(y)$. Assume the contrary; let $m \leq 0$. Let a sequence $\left\{y^{k}\right\} \subset \bar{G} \backslash \mathcal{K}$ be such that $v\left(y^{k}\right) \rightarrow m$ as $k \rightarrow \infty$. Since the sequence $\left\{y^{k}\right\}$ is bounded, it 
contains a convergent subsequence (which we also denote by $\left\{y^{k}\right\}$ ). Let $y^{k} \rightarrow y^{0}$ as $k \rightarrow \infty$, where $y^{0} \in \bar{G}$.

Using the maximum principle, the nonlocal conditions in (4.1), and relations (2.3), one can verify that $y_{0} \notin \bar{G} \backslash \mathcal{K}$. Assume that $y^{0} \in \mathcal{K}_{\nu}$ for some $\nu$. It follows from what has been proved in item 1 that there is a constant $A>0$ such that $w^{1}(y) \geq A$ in some neighborhood of $y^{0}$ (excluding the point $y^{0}$ itself, at which the function $w^{1}$ need not be defined). On the other hand, we have proved in item 3 that $v^{1}\left(y^{0}\right)=0$. Therefore, $v(y) \geq A / 2$ in some neighborhood of $y^{0}$ (excluding the point $y^{0}$ itself). Thus, the sequence $\left\{v\left(y^{k}\right)\right\}$ cannot converge to the nonpositive number $m$.

For any closed set $Q \subset \bar{G}$ such that $Q \cap \mathcal{K} \neq \varnothing$, we introduce the space

$$
C_{\mathcal{K}}(Q)=\{u \in C(Q): u(y)=0, y \in Q \cap \mathcal{K}\}
$$

with the maximum-norm.

Consider the space of vector-valued functions $\mathcal{C}_{\mathcal{K}}(\partial G)=\prod_{i=1}^{N} C_{\mathcal{K}}\left(\overline{\Gamma_{i}}\right)$ with the norm

$$
\|\psi\|_{\mathcal{C}_{\mathcal{K}}(\partial G)}=\max _{i=1, \ldots, N} \max _{y \in \overline{\Gamma_{i}}}\left\|\psi_{i}\right\|_{C\left(\overline{\Gamma_{i}}\right)}
$$

where $\psi=\left\{\psi_{i}\right\}, \psi_{i} \in C_{\mathcal{K}}\left(\overline{\Gamma_{i}}\right)$.

We study the solvability of the problem

$$
P u-q u=0, y \in G ;\left.\quad u\right|_{\Gamma_{i}}-\mathbf{B}_{i} u=\psi_{i}(y), y \in \Gamma_{i}, i=1, \ldots, N,
$$

in the space of continuous functions.

Lemma 4.2. Let Conditions 2.12 .3 be fulfilled, and let $q \geq q_{1}$. Then, for any $\psi=\left\{\psi_{i}\right\} \in \mathcal{C}_{\mathcal{K}}(\partial G)$, there exists a unique solution $u \in C_{\mathcal{K}}(\bar{G}) \cap C^{\infty}(G)$ of problem (4.13). Furthermore, the following estimate holds:

$$
\|u\|_{C(\bar{G})} \leq c_{1}\|\psi\|_{\mathcal{C}_{\mathcal{K}}(\partial G)},
$$

where $c_{1}>0$ does not depend on $\psi$ and $q$.

Proof. 1. We prove the lemma for the functions $\psi_{i}$ which are infinitely differentiable and vanish in a neighborhood of the sets $\overline{\Gamma_{i}} \cap \mathcal{K}$. The general case will follow by the limit passage. Given $\psi_{i}$ with the above properties, we have $\psi_{i} \in H_{-\delta}^{3 / 2}\left(\Gamma_{i}\right)$. Therefore, by Theorem 2.1, there exists a unique solution $u \in H_{1-\delta}^{2}(G)$ of problem (4.13). By Lemma 5.1 in [13], $u \in C^{\infty}(\bar{G} \backslash \mathcal{K})$. Let $\left\{\lambda_{k}\right\}$ be a (finite) set of eigenvalues of $\tilde{\mathcal{L}}(\lambda)$ lying in the strip $-1-\delta<\operatorname{Im} \lambda<-\delta$. Then, due to Theorem 2.2 in [3] (about the asymptotics of solutions for nonlocal problems), the function $u$ has the following asymptotics near an arbitrary point $g_{j} \in \mathcal{K}_{\nu}\left(j=1, \ldots, N_{\nu}, \nu=1, \ldots, N_{0}\right)$ :

$$
u(y)=\sum_{k} \sum_{q=1}^{J_{k}} \sum_{m=0}^{\varkappa_{q k}-1} c_{k}^{(m, q)} W_{k j}^{(m, q)}+u^{\prime}(y), \quad y \in G \cap \mathcal{O}_{\varepsilon}\left(g_{j}\right),
$$

where $c_{k}^{(m, q)}$ are constants, the functions $W_{k j}^{(m, q)}(\omega, r)$ are of the same form as the components of the vector $W_{k}^{(m, q)}(\omega, r)$ in (3.9) $)\left(\omega, r\right.$ are the polar coordinate centered at the point $\left.g_{j}\right)$, and $u^{\prime} \in H_{-\delta}^{2}(G)$. Therefore, applying the Sobolev embedding theorem, we see that $u \in C(\bar{G})$ and

$$
u(y)=0, \quad y \in \mathcal{K} .
$$


2. Let us prove estimate (4.14). Set $M=\|\psi\|_{\mathcal{C}_{\mathcal{K}}(\partial G)}$ and assume that $M>0$.

Set $w_{ \pm}(y)=M v(y) \pm u(y)$, where $v(y)$ is the function from Lemma 4.1. Equalities (4.1) and (4.13) imply that the functions $w_{ \pm}$satisfy the relations

$$
P w_{ \pm}-q w_{ \pm}=M\left(q_{1}-q\right) v(y), y \in G ;\left.\quad w_{ \pm}\right|_{\Gamma_{i}}-\mathbf{B}_{i} w_{ \pm}=M \pm \psi_{i}(y), y \in \Gamma_{i}, i=1, \ldots, N .
$$

Since $q_{1} \leq q, v(y)>0, y \in G$ (by Lemma 4.1), and $M \geq \pm \psi_{i}$, it follows that

$$
P w_{ \pm}-q w_{ \pm} \leq 0, y \in G,\left.\quad w_{ \pm}\right|_{\Gamma_{i}}-\mathbf{B}_{i} w_{ \pm} \geq 0, y \in \Gamma_{i}, i=1, \ldots, N
$$

We claim that $m_{ \pm}=\inf _{y \in \bar{G} \backslash \mathcal{K}} w_{ \pm}(y) \geq 0$. Assume the contrary; let $m_{ \pm}<0$. As in item 4 of the proof of Lemma 4.1, we consider a sequence $\left\{y^{k}\right\} \subset \bar{G} \backslash \mathcal{K}$ such that $y^{k} \rightarrow y^{0}$ and $w_{ \pm}\left(y_{k}\right) \rightarrow m_{ \pm}$ as $k \rightarrow \infty$, where $y^{0} \in \bar{G}$. The following three cases are possible: $y^{0} \in G, y^{0} \in \Gamma_{i}$ for some $i$, and $y^{0} \in \mathcal{K}$.

Let $y^{0} \in G$. Since $w_{ \pm}(y)$ is continuous in $G$, we see that it achieves its negative minimum $m$ inside the domain. It follows from the first inequality in (4.16) and from the maximum principle that $w_{ \pm}(y)=m_{ \pm}$for $y \in G$. Combining this relation with Condition 2.1, we obtain $P w_{ \pm}\left(y^{0}\right)-q w_{ \pm}\left(y^{0}\right)=$ $p_{0}\left(y^{0}\right) m_{ \pm}-q m_{ \pm} \geq-q m_{ \pm}>0$, which contradicts the first inequality in (4.16).

Let $y^{0} \in \Gamma_{i}$ for some $i$. In this case, it follows from (4.16) and (2.3) that

$$
m_{ \pm}=w_{ \pm}\left(y^{0}\right) \geq \sum_{s=1}^{S_{i}} b_{i s}\left(y^{0}\right) w_{ \pm}\left(\Omega_{i s}\left(y^{0}\right)\right) \geq m_{ \pm} \sum_{s=1}^{S_{i}} b_{i s}\left(y^{0}\right) \geq m_{ \pm} .
$$

Therefore, the inequalities in (4.17) are in fact equalities. This means that $\sum_{s=1}^{S_{i}} b_{i s}\left(y^{0}\right)=1$ and $w_{ \pm}\left(\Omega_{i s}\left(y^{0}\right)\right)=m_{ \pm}$for some $s$, i.e., the function $w_{ \pm}(y)$ achieves its negative minimum at the interior point $\Omega_{i s}\left(y^{0}\right) \in G$. This contradicts what has been proved above.

Finally, assume that $y^{0} \in \mathcal{K}_{\nu}$ for some $\nu$. By Lemma 4.1 we have $m=\inf _{y^{\prime} \in \bar{G} \backslash \mathcal{K}} v\left(y^{\prime}\right)>0$, which yields

$$
M v(y) \geq M m>0, \quad y \in \bar{G} \backslash \mathcal{K} .
$$

It follows from the latter inequality and from (4.15) that

$$
w_{ \pm}(y)=M v(y) \pm u(y) \geq M m / 2>0
$$

in some neighborhood of $y^{0}$ (excluding the point $y^{0}$ itself, where $w_{ \pm}(y)$ need not be defined). Therefore, the sequence $\left\{w_{ \pm}\left(y^{k}\right)\right\}$ cannot converge to the negative number $m_{ \pm}$.

Thus, we have proved that $\inf _{y \in \bar{G} \backslash \mathcal{K}} w_{ \pm}(y) \geq 0$, which yields

$$
|u(y)| \leq M v(y) \leq M \sup _{y^{\prime} \in \bar{G} \backslash \mathcal{K}} v\left(y^{\prime}\right), \quad y \in \bar{G} \backslash \mathcal{K} .
$$

Since the function $u(y)$ is continuous in $\bar{G}$, the last inequality implies estimate (4.14), where $c_{1}=$ $\sup v\left(y^{\prime}\right)$. Clearly, the constant $c_{1}>0$ does not depend on $\psi$ and $q$. $y^{\prime} \in \bar{G} \backslash \mathcal{K}$

Now we consider the problem

$$
P u-q u=f(y), y \in G ;\left.\quad u\right|_{\Gamma_{i}}-\mathbf{B}_{i} u=\psi_{i}(y), y \in \Gamma_{i}, i=1, \ldots, N .
$$


Theorem 4.1. Let Conditions 2.12 .3 be fulfilled, and let $q \geq q_{1}$. Then, for any $f \in C(\bar{G})$ and $\psi=\left\{\psi_{i}\right\} \in \mathcal{C}_{\mathcal{K}}(\partial G)$, there exists a unique solution $u \in C_{\mathcal{K}}(\bar{G}) \cap W_{2, \text { loc }}^{2}(G)$ of problem (4.18). Furthermore, if $f=0$, then $u \in C_{\mathcal{K}}(\bar{G}) \cap C^{\infty}(G)$ and the following estimate holds:

$$
\|u\|_{C(\bar{G})} \leq c_{1}\|\psi\|_{\mathcal{C}_{\mathcal{K}}(\partial G)},
$$

where $c_{1}>0$ does not depend on $\psi$ and $q$.

Proof. Due to Lemma 4.2, it suffices to prove the existence of a solution $u \in C_{\mathcal{K}}(\bar{G}) \cap W_{2, \text { loc }}^{2}(G)$ for problem (4.18) with $f \in C(\bar{G})$ and $\psi_{i}=0$. Since $f \in C(\bar{G}) \subset H_{-\delta}^{0}(G)$, it follows from Theorem 2.1 that there is a unique solution $u \in H_{1-\delta}^{2}(G)$ of problem (4.18) with the right-hand sides $\psi_{i}=0$. By Lemma 5.1 in [13] $u \in W_{2}^{2}\left(G \backslash \overline{\mathcal{O}_{\sigma}(\mathcal{K})}\right)$ for all $\sigma>0$.

Let $\left\{\lambda_{k}\right\}$ be a (finite) set of eigenvalues of $\tilde{\mathcal{L}}(\lambda)$ lying in the strip $-1-\delta<\operatorname{Im} \lambda<-\delta$. Then, due to Theorem 2.2 in [3] (about the asymptotics of solutions for nonlocal problems), the function $u$ has the following asymptotics near an arbitrary point $g_{j} \in \mathcal{K}_{\nu}\left(j=1, \ldots, N_{\nu}, \nu=1, \ldots, N_{0}\right)$ :

$$
u(y)=\sum_{k} \sum_{q=1}^{J_{k}} \sum_{m=0}^{\varkappa_{q k}-1} c_{k}^{(m, q)} W_{k j}^{(m, q)}+u^{\prime}(y), \quad y \in G \cap \mathcal{O}_{\varepsilon}\left(g_{j}\right),
$$

where $c_{k}^{(m, q)}$ are constants, the functions $W_{k j}^{(m, q)}(\omega, r)$ are of the same form as the components of the vector $W_{k}^{(m, q)}(\omega, r)$ in (3.9) $\left(\omega, r\right.$ are the polar coordinate centered at the point $\left.g_{j}\right)$, and $u^{\prime} \in H_{-\delta}^{2}(G)$. Therefore, applying the Sobolev embedding theorem, we see that $u \in C_{\mathcal{K}}(\bar{G})$.

\section{$5 \quad$ Existence of Feller semigroups}

We introduce the space

$$
C_{B}(\bar{G})=\left\{u \in C_{\mathcal{K}}(\bar{G}):\left.u\right|_{\Gamma_{i}}-\mathbf{B}_{i} u=0, y \in \Gamma_{i}, i=1, \ldots, N\right\} .
$$

We prove in this section that the unbounded operator $\mathbf{P}_{B}: \mathrm{D}\left(\mathbf{P}_{B}\right) \subset C_{B}(\bar{G}) \rightarrow C_{B}(\bar{G})$ given by

$$
\mathbf{P}_{B} u=P u, \quad \mathrm{D}\left(\mathbf{P}_{B}\right)=\left\{u \in C_{B}(\bar{G}) \cap W_{2, \text { loc }}^{2}(G): P u \in C_{B}(\bar{G})\right\},
$$

is a generator of a Feller semigroup.

Remark 5.1. Consider a nontransversal nonlocal condition of the form (cf. [1, 2, 6, 11, 16, 18])

$$
b(y) u(y)+\int_{\bar{G}}[u(x)-u(\eta)] m(y, d \eta)=0, \quad y \in \partial G,
$$

where $b(y) \geq 0, m(y, \cdot)$ is a nonnegative Borel measure, and $b(y)+m(y, \bar{G})>0, y \in \partial G$.

Introduce a nonnegative Borel measure $\mu(y, \cdot)=m(y, \cdot) /[b(y)+m(y, \bar{G})]$. Then the nonlocal condition (5.2) can be written as follows:

$$
u(y)-\int_{\bar{G}} u(\eta) \mu(y, d \eta)=0, \quad y \in \partial G .
$$

Assume that $\mu(y, \cdot)=0$ for $y \in \mathcal{K}$ and $\mu(y, \cdot)$ is a linear combination of delta-functions, supported at the points $\Omega_{i s}(y)$, with the coefficients $b_{i s}(y)$ for $y \in \Gamma_{i}$. Then the nonlocal conditions (5.3) and (5.2) assume the form

$$
\left.u\right|_{\Gamma_{i}}-\mathbf{B}_{i} u=0, y \in \Gamma_{i}, i=1, \ldots, N ; \quad u(y)=0, y \in \mathcal{K} .
$$


Lemma 5.1. Let Conditions $2.1+2.3$ hold. Let a function $u \in C_{B}(\bar{G})$ achieve its positive maximum at a point $y^{0} \in \bar{G}$, and let $P u \in C(G)$. Then there is a point $y^{1} \in G$ such that $u\left(y^{1}\right)=u\left(y^{0}\right)$ and $P u\left(y^{1}\right) \leq 0$.

Proof. If $y^{0} \in G$, then the conclusion of the lemma follows from the maximum principle. Let $y^{0} \in \partial G$. Assume that the lemma is not true, i.e., $u\left(y^{0}\right)>u(y)$ for all $y \in G$.

Since $u\left(y^{0}\right)>0$, it follows that $y^{0} \in \Gamma_{i} \cap \mathcal{O}_{\varepsilon}(\mathcal{K})$ for some $i$ and $b_{i s}\left(y^{0}\right)>0$ for some $s$. Taking into account that $\Omega_{i s}\left(y^{0}\right) \in G$ and $u\left(y^{0}\right)>u(y)$ for all $y \in G$, we have $u\left(y^{0}\right)-u\left(\Omega_{i s}\left(y^{0}\right)\right)>0$. Therefore, using (2.3), we obtain

$$
0=u\left(y^{0}\right)-\sum_{s=1}^{S_{i}} b_{i s}\left(y^{0}\right) u\left(\Omega_{i s}\left(y^{0}\right)\right) \geq \sum_{s=1}^{S_{i}} b_{i s}\left(y^{0}\right)\left(u\left(y^{0}\right)-u\left(\Omega_{i s}\left(y^{0}\right)\right)\right)>0 .
$$

The contradiction proves the lemma.

Corollary 5.1. Let Conditions 2.12 .3 hold. Let $u \in C_{B}(\bar{G})$ be a solution of the equation

$$
q u(y)-P u(y)=f(y), \quad y \in G
$$

where $q>0$ and $f \in C(\bar{G})$. Then

$$
\|u\|_{C(\bar{G})} \leq \frac{1}{q}\|f\|_{C(\bar{G})}
$$

Proof. Let $\max _{y \in \bar{G}}|u(y)|=u\left(y^{0}\right)>0$ for some $y^{0} \in \bar{G}$. Then, by Lemma $\left[5.1\right.$, there is a point $y^{1} \in G$ such that $u\left(y^{1}\right)=u\left(y^{0}\right)$ and $P u\left(y^{1}\right) \leq 0$. Hence,

$$
\|u\|_{C(\bar{G})}=u\left(y^{0}\right)=u\left(y^{1}\right)=\frac{1}{q}\left(P u\left(y^{1}\right)+f\left(y^{1}\right)\right) \leq \frac{1}{q}\|f\|_{C(\bar{G})} .
$$

Lemma 5.2. Let Conditions 2.12 .3 hold. Then $\mathrm{D}\left(\mathbf{P}_{B}\right)$ is dense in $C_{B}(\bar{G})$.

Proof. We will follow the scheme proposed in [11].

1. Let $u \in C_{B}(\bar{G})$. Since $C_{B}(\bar{G}) \subset C_{\mathcal{K}}(\bar{G})$, it follows that, for any $\varepsilon>0$ and $q \geq q_{1}$, there is a function $u_{1} \in C^{\infty}(\bar{G}) \cap C_{\mathcal{K}}(\bar{G})$ such that

$$
\left\|u-u_{1}\right\|_{C(\bar{G})} \leq \min \left(\varepsilon, \varepsilon /\left(2 c_{1}\right)\right)
$$

where $c_{1}$ is the number from Lemma 4.2 .

Set

$$
\begin{array}{rlrl}
f(y) & \equiv q u_{1}-P u_{1}, & y & \in G, \\
\psi_{i}(y) & \equiv u_{1}(y)-\mathbf{B}_{i} u_{1}(y), \quad y \in \Gamma_{i}, i=1, \ldots, N .
\end{array}
$$

Since $u_{1} \in C_{\mathcal{K}}(\bar{G})$, it follows that $\left\{\psi_{i}\right\} \in \mathcal{C}_{\mathcal{K}}(\partial G)$. Using the relation

$$
u(y)-\mathbf{B}_{i} u(y)=0, \quad y \in \Gamma_{i},
$$

inequality (5.5), and relations (2.3), we obtain

$$
\left\|\left\{\psi_{i}\right\}\right\|_{\mathcal{C}_{\mathcal{K}}(\partial G)} \leq\left\|u-u_{1}\right\|_{C(\bar{G})}+\left\|\left\{\mathbf{B}_{i}\left(u-u_{1}\right)\right\}\right\|_{\mathcal{C}_{\mathcal{K}}(\partial G)} \leq \varepsilon / c_{1} .
$$


Consider the auxiliary nonlocal problem

$$
\begin{gathered}
q u_{2}-P u_{2}=f(y), \quad y \in G, \\
u_{2}(y)-\mathbf{B}_{i} u_{2}(y)=0, y \in \Gamma_{i} ; \quad u_{2}(y)=0, y \in \mathcal{K} .
\end{gathered}
$$

Since $f \in C^{\infty}(\bar{G})$, it follows from Theorem 4.1 that problem (5.8) has a unique solution $u_{2} \in C_{B}(\bar{G})$.

Using (5.6), (5.8), and the relations $u_{1}(y)=u_{2}(y)=0, y \in \mathcal{K}$, we see that the function $w_{1}=$ $u_{1}-u_{2}$ satisfies the relations

$$
\begin{gathered}
q w_{1}-P w_{1}=0, \quad y \in G, \\
w_{1}(y)-\mathbf{B}_{i} w_{1}(y)=\psi_{i}(y), y \in \Gamma_{i} ; \quad w_{1}(y)=0, y \in \mathcal{K} .
\end{gathered}
$$

By Lemma 4.2, problem (5.9) has a unique solution $w_{1} \in C_{B}(\bar{G})$ and (taking (5.7) into account)

$$
\left\|w_{1}\right\|_{C(\bar{G})} \leq c_{1}\left\|\left\{\psi_{i}\right\}\right\|_{\mathcal{C}_{\mathcal{K}}(\partial G)} \leq c_{1} \varepsilon / c_{1}=\varepsilon .
$$

2. Finally, we consider the problem

$$
\begin{aligned}
\lambda u_{3} & -P u_{3}=\lambda u_{2}, \quad y \in G, \\
u_{3}(y)-\mathbf{B}_{i} u_{3}(y) & =0, y \in \Gamma_{i} ; \quad u_{3}(y)=0, y \in \mathcal{K} .
\end{aligned}
$$

Since $u_{2} \in C_{B}(\bar{G})$, it follows from Theorem 4.1 that problem (5.11) has a unique solution $u_{3} \in \mathrm{D}\left(\mathbf{P}_{B}\right)$ for all sufficiently large $\lambda>0$.

Denote $w_{2}=u_{2}-u_{3} \in C_{B}(\bar{G})$. It follows from (5.11) that

$$
\lambda w_{2}-P w_{2}=-P u_{2}=f-q u_{2} .
$$

Applying Corollary 5.1, we have

$$
\left\|w_{2}\right\|_{C(\bar{G})} \leq \frac{1}{\lambda}\left\|f-q u_{2}\right\|_{C(\bar{G})} .
$$

Choosing sufficiently large $\lambda$ yields

$$
\left\|w_{2}\right\|_{C(\bar{G})} \leq \varepsilon
$$

Inequalities (5.5), (5.10), and (5.12) imply

$$
\left\|u-u_{3}\right\|_{C(\bar{G})} \leq\left\|u-u_{1}\right\|_{C(\bar{G})}+\left\|u_{1}-u_{2}\right\|_{C(\bar{G})}+\left\|u_{2}-u_{3}\right\|_{C(\bar{G})} \leq 3 \varepsilon .
$$

Now we can prove the main result about the existence of a Feller semigroup.

Theorem 5.1. Let conditions 2.12 .3 hold. Then the operator $\mathbf{P}_{B}: \mathrm{D}\left(\mathbf{P}_{B}\right) \subset C_{B}(\bar{G}) \rightarrow C_{B}(\bar{G})$ is a generator of a Feller semigroup.

Proof. 1. By Lemma 5.2, the domain of the operator $\mathbf{P}_{B}$ is dense in $C_{B}(\bar{G})$.

2. By Theorem 4.1 and Corollary [5.1, there exists a bounded operator $\left(q I-\mathbf{P}_{B}\right)^{-1}: C_{B}(\bar{G}) \rightarrow$ $C_{B}(\bar{G})$ for all sufficiently large $q>0$ and

$$
\left\|\left(q I-\mathbf{P}_{B}\right)^{-1}\right\| \leq 1 / q .
$$

3. Let us prove that the operator $\left(q I-\mathbf{P}_{B}\right)^{-1}$ is nonnegative. Assume the contrary. Then there is a function $f \geq 0$ such that the solution $u \in \mathrm{D}\left(\mathbf{P}_{B}\right)$ of the equation $q u-\mathbf{P}_{B} u=f$ achieves its 
negative minimum at some point $y^{0} \in \bar{G}$. Therefore, the function $v=-u$ achieves its positive maximum at the point $y^{0}$. Due to Lemma 5.1, there exists a point $y^{1} \in G$ such that $v\left(y^{1}\right)=v\left(y^{0}\right)$ and $\mathbf{P}_{B} v\left(y^{1}\right) \leq 0$. Hence, $0<v\left(y^{0}\right)=v\left(y^{1}\right)=\left(\mathbf{P}_{B} v\left(y^{1}\right)-f\left(y^{1}\right)\right) / q \leq 0$. This contradiction shows that $u \geq 0$.

Thus all the hypotheses of the Hille-Iosida theorem (Theorem 1.1) hold, and the operator $\mathbf{P}_{B}$ : $\mathrm{D}\left(\mathbf{P}_{B}\right) \subset C_{B}(\bar{G}) \rightarrow C_{B}(\bar{G})$ is a generator of a Feller semigroup.

The author is grateful to Prof. A.L. Skubachevskii for attention to this work.

The work was supported by Russian Foundation for Basic Research (project No. 07-01-00268) and the Alexander von Humboldt Foundation.

\section{References}

[1] A. D. Ventsel, "On boundary conditions for multidimensional diffusion processes," Teor. Veroyatnost. i Primen., 4, 172-185 (1959); English transl.: Theory Probab. Appl., 4 (1959).

[2] E. I. Galakhov and A. L. Skubachevskii, "On contractive nonnegative semigroups with nonlocal conditions," Mat. Sb., 189, 45-78 (1998); English transl.: Math. Sb. 189 (1998).

[3] P. L. Gurevich, "Asymptotics of solutions for nonlocal elliptic problems in plane angles," Trudy seminara imeni I. G. Petrovskogo, 23, 93-126 (2003); English transl.: J. Math. Sci., 120, No. 3, 1295-1312 (2004).

[4] P. L. Gurevich and A. L. Skubachevskii, "On the fredholm and unique solvability of nonlocal elliptic problems in multidimensional domains," Trudy Moskov. Mat. Obshch., 268, 288-373 (2007); English transl.: Trans. Moscow Math. Soc.

[5] S. A. Nazarov and B. A. Plamenevskii, Elliptic Problems in Domains with Piecewise Smooth Boundaries, De Gruyter Expositions in Mathematics, 13. Walter de Gruyter Publichers, Berlin - New York, 1994.

[6] A. L. Skubachevskii, "On some problems for multidimensional diffusion processes," Dokl. Akad. Nauk SSSR, 307, 287-291 (1989); English transl.: Soviet Math. Dokl. 40 (1990).

[7] A. L. Skubachevskii, "Model nonlocal problems for elliptic equations in dihedral angles," Differentsial'nye Uravneniya, 26, 119-131 (1990); English transl.: Differ. Equ., 26 (1990).

[8] J. M. Bony, P. Courrege, and P. Priouret, "Semi-groups de Feller sur une variété à bord compacte et problèmes aux limites intégro-différentiels du second ordre donnant lieu au principe du maximum," Ann. Inst. Fourier (Grenoble), 18, 369-521 (1968).

[9] W. Feller, "The parabolic differential equations and the associated semi-groups of transformations," Ann. Math., 55, 468-519 (1952).

[10] W. Feller, "Diffusion processes in one dimension," Trans. Amer. Math. Soc., 77, 1-30 (1954).

[11] E. I. Galakhov and A. L. Skubachevskii, "On Feller semigroups generated by elliptic operators with integro-differential boundary conditions," J. Differential Equations, 176, 315-355 (2001).

[12] P. L. Gurevich, "Nonlocal problems for elliptic equations in dihedral angles and the Green formula," In Mitteilungen aus dem Mathem. Seminar Giessen, Math. Inst. Univ. Giessen, Germany, 247, 1-74 (2001). 
[13] P. L. Gurevich, "Solvability of nonlocal elliptic problems in Sobolev spaces, II," Russ. J. Math. Phys., 11, No. 1, 1-44 (2004).

[14] Y. Ishikawa, "A remark on the existence of a diffusion process with non-local boundary conditions" J. Math. Soc. Japan, 42, 171-184 (1990).

[15] K. Sato and T. Ueno, "Multi-dimensional diffusion and the Markov process on the boundary," J. Math. Kyoto Univ., 4, 529-605 (1965).

[16] A. L. Skubachevskii, "Nonlocal elliptic problems and multidimensional diffusion processes" Russian J. Mathematical Physics, 3, 327-360 (1995).

[17] K. Taira, Diffusion Processes and Partial Differential Equations. New York — London: Academic Press, 1988.

[18] K. Taira, Semigroups, boundary value problems and Markov processes. Berlin: Springer-Verlag, 2004.

[19] S. Watanabe, "Construction of diffusion processes with Wentzell's boundary conditions by means of Poisson point processes of Brownian excursions," In: Probability Theory, 5, 255-271 (1979). Banach Center Publications. Warsaw: Polish Scientific Publishers, 1979. 